\title{
Hyperthyroidism, CTCAE
}

National Cancer Institute

\section{Source}

National Cancer Institute. Hyperthyroidism, CT CAE. NCI Thesaurus. Code C143560.

A disorder characterized by excessive levels of thyroid hormone in the body. Common causes include an overactive thyroid gland or thyroid hormone overdose. 